\title{
SOME ONE-SIDED THEOREMS ON THE TAIL DISTRIBUTION OF SAMPLE SUMS WITH APPLICATIONS TO THE LAST TIME AND LARGEST EXCESS OF BOUNDARY CROSSINGS
}

\author{
BY
}

\author{
Y. S. CHOW( $\left.{ }^{1}\right)$ AND T. L. LAI( $\left.{ }^{2}\right)$
}

ABSTRACT. In this paper, we prove certain one-sided Paley-type inequalities and use them to study the convergence rates for the tail probabilities of sample sums. We then apply our results to find the limiting moments and the limiting distribution of the last time and the largest excess of boundary crossings for the sample sums, generalizing the results previously obtained by Robbins, Siegmund and Wendel. Certain one-sided limit theorems for delayed sums are also obtained and are applied to study the convergence rates of tail probabilities.

1. Introduction. Let $X_{1}, X_{2}, \ldots$ be i.i.d. random variables, and let $S_{n}$ $=X_{1}+\cdots+X_{n}, S_{0}=X_{0}=0$. If $E X_{1}=0$, then for any $\epsilon>0, P\left[\left|S_{n}\right| \geq \epsilon n\right]$ converges to 0 as $n \rightarrow \infty$. The rate at which the above convergence takes place, and more generally, the rate of convergence for $P\left[\left|S_{n}\right| \geq \epsilon n^{a}\right], a>1 / 2$, have been studied by a number of authors. In [1], Baum and Katz have proved that for any $p>1 / \alpha, a>1 / 2$, the following statements are equivalent:

$$
\begin{gathered}
\sum n^{p a-2} P\left[\left|S_{n}\right| \geq \epsilon n^{a}\right]<\infty \text { for all } \epsilon>0, \\
\sum n^{p a-2} P\left[\sup _{k \geq n}\left|S_{k} / k^{a}\right| \geq \epsilon\right]<\infty \text { for all } \epsilon>0, \\
E\left|X_{1}\right|^{p}<\infty, \text { and for the case } a \leq 1, \quad E X_{1}=0 .
\end{gathered}
$$

The analogous situation corresponding to the limiting case $\alpha=1 / 2$ has been considered in [11], where it is proved that, for any $p>2$, the following statements are equivalent:

Received by the editors December 12, 1973.

AMS (MOS) subject classifications (1970). Primary 60F 10, 60F 15, 60G50.

Key words and phrases. Convergence rates, Paley-type inequalities, last time, largest excess, limiting distribution, limiting moments, delayed sums.

(1) Research supported by the National Science Foundation under Grant NSF-GP. 33570X at Columbia University.

(2) Research supported by the Office of Naval Research under Contract No. N00014-67-A-0108-0018 at Columbia University. 


$$
\begin{gathered}
E\left|X_{1}\right|^{p}\left(\log ^{+}\left|X_{1}\right|+1\right)^{-p / 2}<\infty \quad \text { and } E X_{1}=0, \\
\sum n^{p / 2-2} P\left[\left|S_{n}\right| \geq \epsilon(n \log n)^{1 / 2}\right]<\infty \quad \text { for all large } \epsilon, \\
\sum n^{p / 2-2} P\left[\sup _{k \geq n}\left|S_{k} /(k \log k)^{1 / 2}\right| \geq \epsilon\right]<\infty \quad \text { for all large } \epsilon .
\end{gathered}
$$

It is natural to ask whether there are corresponding one-sided analogues of the above results. For example, if $1 / 2<\alpha<1$ and $X_{1}, X_{2}, \ldots$ are i.i.d. random variables with $E X_{1}=0$ and $E\left(X_{1}^{\dagger}\right)^{p}<\infty$ for some $p>1 / a$, then is it always true that $\Sigma n^{p a-1} P\left[S_{n} \geq \epsilon n^{a}\right]<\infty$ for all $\epsilon>0$ ? The answer to this question turns out to be negative, as will be shown in $\$ 2$ by a counterexample. However, if we also assume that $E X_{1}^{2}<\infty$, then the answer becomes affirmative. In fact, the following result has been established in [2]. Let $E\left|X_{1}\right|^{r}<\infty$ for some $1 \leq r \leq 2, E\left(X_{1}^{+}\right)^{p}<\infty$ for some $p \geq r$ and $E X_{1}=0$. If $a_{r}>1$, then

$$
\sum n^{p a-2} P\left[\max _{1 \leq k \leq n} S_{k}>\epsilon n^{a}\right]<\infty \text { for all } \epsilon>0 .
$$

The additional requirement $E\left|X_{1}\right|^{r}<\infty$ is a natural assumption, for without it, $P\left[S_{n} \geq \epsilon n^{\alpha}\right]$ may even converge to 1 under $E X_{1}=0$ and $E\left(X_{1}^{+}\right)^{p}<\infty$ for all $p$, as our counterexample shows. In $\$ 3$, we shall obtain a sharper version of (1.7). A corresponding one-sided analogue of (1.5) and (1.6) under the assumption $E X_{1}=0, E X_{1}^{2}<\infty$ and $E\left(X_{1}^{+}\right)^{p}\left(\log \left(2+X_{1}^{+}\right)\right)^{-p / 2}<\infty$ will also be given in $\$ 4$.

The series considered in (1.7) is closely related to the moments of the last time and of the largest excess of certain boundary crossings for the sequence $X_{n}$ and for the sequence of partial sums $S_{n}$. More specifically, let us define

$$
\begin{aligned}
& T(\epsilon, \alpha)=\sup \left\{n \geq 1: S_{n} \geq \epsilon n^{\alpha}\right\} \quad(\sup \phi=0), \\
& M(\epsilon, \alpha)=\sup _{n \geq 0}\left(S_{n}-\epsilon n^{\alpha}\right), \\
& T_{1}(\epsilon, \alpha)=\sup \left\{n \geq 1: X_{n} \geq \epsilon n^{\alpha}\right\}, \\
& M_{1}(\epsilon, \alpha)=\sup _{n \geq 0}\left(X_{n}-\epsilon n^{\alpha}\right) .
\end{aligned}
$$

In $\$ 5$, we shall consider the relations between the series in (1.7) and $E(T(\epsilon, \alpha))^{p a-1}, E\left(T_{1}(\epsilon, \alpha)\right)^{p a-1}, E(M(\epsilon, \alpha))^{(p a-1) / a}$ and $E\left(M_{1}(\epsilon, \alpha)\right)^{(p a-1) / a}$. Our results here extend those found in [3], [8], [9] and [15]. 
In $\$ 3$, to sharpen the relation (1.7), we shall prove the following inequality: If $E X_{1}=0$ and $E X_{1}^{2}<\infty$, then for $\alpha>1 / 2$ and $p>1 / \alpha$,

(1.9) $\sum_{n=1}^{\infty} n^{p a-2} P\left[\max _{1 \leq k<n} S_{k} \geq n^{a}\right] \leq C_{p, a}\left\{E\left(X_{1}^{+}\right)^{p}+\left(E X_{1}^{2}\right)^{(p a-1) /(2 a-1)}\right\}$,

where $C_{p, a}$ is a universal constant depending only on $p$ and $a$. In fact, we shall derive a slightly more general inequality where we consider $E\left|X_{1}\right|^{r}$ in place of $E X_{1}^{2}$ for some $1 \leq r \leq 2$. The inequality (1.9) has some interesting applications in connection with the last time $T(\epsilon, \alpha)$ and the largest excess $M(\epsilon, \alpha)$ of boundary crossings. In $\S 6$, we shall show that as $\epsilon\rfloor 0$,

$$
\begin{aligned}
& \epsilon^{2 /(2 a-1)} T(\epsilon, \alpha) \stackrel{D}{\longrightarrow} T^{*}(\alpha), \\
& \epsilon^{1 /(2 a-1)} M(\epsilon, \alpha) \stackrel{D}{\longrightarrow} M^{*}(\alpha),
\end{aligned}
$$

where "P D" denotes convergence in distribution, and

$$
T^{*}(\alpha)=\sup \left\{t \geq 0: W(t) \geq t^{\alpha}\right\}, \quad M^{*}(\alpha)=\sup _{t \geq 0}\left(W(t)-t^{\alpha}\right)
$$

and $W(t), t \geq 0$, is the standard Wiener process. Using the inequality (1.9), we easily obtain that if $E\left(X_{1}^{+}\right)^{p}<\infty$ for some $p>2$, then

$$
\begin{gathered}
\lim _{\epsilon \downarrow 0} \epsilon^{2(p a-1) /(2 a-1)} E(T(\epsilon, \alpha))^{p a-1}=E\left(T^{*}(\alpha)\right)^{p a-1}, \\
\lim _{\epsilon \downarrow 0} \epsilon^{(p a-1) /\{a(2 a-1)\}} E(M(\epsilon, \alpha))^{(p a-1) / a}=E\left(M^{*}(\alpha)\right)^{(p a-1) / a} .
\end{gathered}
$$

The inequality (1.9) enables us to simplify the proof and extend the result of Robbins, Siegmund and Wendel [15] and Kao [8] who in connection with certain statistical applications have considered the limiting relations (1.10), (1.11), (1.12) and (1.13) in the case $a=1$.

The one-sided inequality (1.9) obviously implies the corresponding twosided result: If $E X_{1}=0, E X_{1}^{2}<\infty, \alpha>1 / 2$ and $p \geq 2$, then

(1.14) $\sum_{n=1}^{\infty} n^{p a-2} P\left[\max _{1 \leq k \leq n}\left|S_{k}\right| \geq n^{a}\right] \leq C_{p, a}\left\{E\left|X_{1}\right|^{p}+\left(E X_{1}^{2}\right)^{(p a-1) /(2 a-1)}\right\}$.

The above upper bound is sharp in the sense that a corresponding lower bound also holds:

$$
\text { (1.15) } 1+\sum_{n=1}^{\infty} n^{p a-2} P\left[\left|S_{n}\right|>n^{\alpha}\right] \geq B_{p, a}\left\{E\left|X_{1}\right|^{p}+\left(E X_{1}^{2}\right)^{(p a-1) /(2 a-1)}\right\} \text {. }
$$

We shall refer to the inequalities (1.14) and (1.15) as Paley-type inequalities because of their resemblance to Paley's theorem which connects the type of integrability of a function with the rate of convergence of its Fourier coeffi- 
cients (cf. [17, Vol. 2, p. 121]). The proof of (1.15) together with other related results and applications will be presented in another paper.

2. A counterexample. Let $0<\delta<2$ and define $\psi(x)=|x|^{-2}(\log |x|)^{-1-\delta}$ for $x \leq-c$, and let $a, b, c$ be positive numbers such that $c \geq e$ and

$$
a \int_{-\infty}^{-c} \psi(x) d x+b=1, \quad a \int_{-\infty}^{-c} x \psi(x) d x+b=0 .
$$

Suppose $X_{1}, X_{2}, \ldots$ are i.i.d. random variables with $P\left[X_{1}=1\right]=b$ and $P\left[X_{1} \leq x\right]=a \int_{-\infty}^{x} \psi(x) d x$ for $x \leq-c$. Then $E X_{1}=0$ and $E\left(X_{1}^{+}\right)^{p}<\infty$ for all $p \geq 1$. Let $X_{n}^{\prime}=X_{n} I_{\left[X_{n} \geq-n(\log n)^{-\delta / 2}\right]}, s_{n}^{\prime}=X_{1}^{\prime}+\cdots+X_{n}^{\prime}$. It is easy to see that

$$
\begin{gathered}
P\left[X_{n} \neq X_{n}^{\prime} \text { i.o. }\right]=0, \\
E S_{n}^{\prime} \sim(a / \delta)_{n}(\log n)^{-\delta} \text { as } n \rightarrow \infty, \\
\sigma\left(S_{n}^{\prime}\right)=\left(\operatorname{var} S_{n}^{\prime}\right)^{1 / 2} \sim(a / 2)^{1 / 2} n(\log n)^{-1 / 2-3 \delta / 4} \text { as } n \rightarrow \infty .
\end{gathered}
$$

Since $\delta<1 / 2+3 \delta / 4$, it follows from (2.2) and (2.3) that $\sigma\left(S_{n}^{\prime}\right)=o\left(E S_{n}^{\prime}\right)$. Therefore using the Tchebychev inequality, it is easy to see that $S_{n}^{\prime} /\left(E S_{n}^{\prime}\right)$ $\stackrel{P}{\longrightarrow}$ 1. This, together with (2.1), in turn implies that

$$
(\delta / a) S_{n}(\log n)^{\delta} / n \stackrel{P}{\longrightarrow} 1 .
$$

Hence $\lim _{n \rightarrow \infty} P\left[S_{n}>\epsilon n^{a}\right]=1$ for any $1>a>1 / 2$, and so in contrast with (1.1), $\Sigma n^{-1} P\left[S_{n} \geq \epsilon n^{\alpha}\right]=\infty$. It is interesting to note that in the case $\alpha=1$, $\sum n^{-1} P\left[S_{n} \geq \epsilon n\right]<\infty$ for all $\epsilon>0$ by Spitzer's theorem [16].

We remark that our counterexample also gives a negative answer to the following question. The Marcinkiewicz-Zygmund strong law of large numbers states that if $X_{1}, X_{2}, \ldots$ are i.i.d. with $E X_{1}=0$ and $E\left|X_{1}\right|^{p<\infty}$ for some $1 \leq p<2$, then $n^{-1 / p} S_{n} \rightarrow 0$ a.e. It is natural to ask whether the one-sided analogue, i.e., $\lim \sup _{n \rightarrow \infty} n^{-1 / p} S_{n} \leq 0$ a.e., would hold if $E X_{1}=0$ and $E\left(X_{1}^{+}\right)^{p}<\infty$. We note that this fails to hold in our example, since (2.4) implies that

$$
\underset{n \rightarrow \infty}{\lim \sup _{0}}(\delta / a) S_{n}(\log n)^{\delta} / n \geq 1 \text { a.e. }
$$

3. A one-sided Paley-type inequality and its application to the convergence rate of tail probabilities. Let $X_{1}, X_{2}, \ldots$ be a sequence of random variables. Henceforth we shall use the following notation: For any real numbers $t, r \geq 1$, 


$$
S_{t}=\sum_{i=1}^{[t]} X_{i}, \quad \bar{S}_{t}=\max _{1 \leq j \leq t} S_{j}, \quad \bar{X}_{t}=\max _{1 \leq j \leq t} X_{j}
$$

$$
S_{r, t}=\sum_{r<j \leq[r]+t} X_{j}, \quad \bar{S}_{r, t}=\max _{1 \leq j \leq t} S_{r, j}, \quad S_{0}=\bar{S}_{0}=X_{0}=\bar{X}_{0}=0 .
$$

We first prove an inequality of which (1.9) is a special case.

Theorem 1. Suppose $X_{1}, X_{2}, \ldots$ are i.i.d. with $E X_{1}=0$ and $E\left|X_{1}\right|^{r}<\infty$ for some $1 \leq r \leq 2$. Let $\alpha>1 / r$ and $p>1 / \alpha$. Then there exists a universal constant $C_{p, a, r}>0$ depending only on $p, \alpha$ and $r$ such that

$$
\sum_{n=1}^{\infty} n^{p a-2} P\left[\bar{S}_{n} \geq n^{a}\right] \leq C_{p, a, r}\left\{E\left(X_{1}^{+}\right)^{p}+\left(E\left|X_{1}\right|^{r}\right)^{(p a-1) /(r a-1)}\right\} .
$$

Proof. Let $k$ be the smallest positive integer $>(p \alpha-1) /(r \alpha-1)$. We note that

$$
\begin{aligned}
P\left[\bar{S}_{n} \geq n^{a}\right] & \leq P\left[\bar{X}_{n}>n^{a} /(2 k)\right]+P\left[\bar{S}_{n} \geq n^{a}, \bar{X}_{n} \leq n^{a} /(2 k)\right] \\
& \leq n P\left[X_{1}>n^{a} /(2 k)\right]+P\left[\bar{S}_{n} \geq n^{a}, \bar{X}_{n} \leq n^{\alpha} /(2 k)\right] .
\end{aligned}
$$

For each fixed $n$, define

$$
\begin{aligned}
& \tau_{1}=\tau_{1}^{(n)}=\inf \left\{j \geq 1: S_{j} \geq n^{a} /(2 k)\right\}, \\
& \tau_{2}=\tau_{2}^{(n)}=\inf \left\{j \geq 1: S_{\tau_{1}+j}-S_{\tau_{1}} \geq n^{a} /(2 k)\right\}, \text { etc. }
\end{aligned}
$$

Without loss of generality, we can assume that $E\left|X_{1}\right| \neq 0$. Since $E X_{1}=0$, it follows from the Chung-Fuchs theorem that $P\left[\tau_{1}<\infty\right]=1$. Also $\tau_{1}, \tau_{2}, \ldots$ are i.i.d. random variables. Hence

$$
\begin{aligned}
P\left[\bar{S}_{n} \geq n^{\alpha}, \bar{X}_{n} \leq n^{\alpha} /(2 k)\right] & \leq P\left[\tau_{1}+\cdots+\tau_{k} \leq n\right] \\
& \leq P^{k}\left[r_{1} \leq n\right]=P^{k}\left[\bar{S}_{n} \geq n^{\alpha} /(2 k)\right] .
\end{aligned}
$$

Now there exist positive constant $A_{r}$ and $B_{r}$ depending only on $r$ such that

$$
\begin{aligned}
P\left[\bar{S}_{n} \geq n^{\alpha} /(2 k)\right] & \leq A_{r}(2 k)^{r} n^{-r a} E\left|S_{n}\right|^{r} \quad(\text { cf. [4, p. 317]) } \\
& \leq B_{r}(2 k)^{r} n^{-(r a-1)} E\left|X_{1}\right|^{r} .
\end{aligned}
$$

The last inequality above follows from the Marcinkiewicz-Zygmund inequalities (cf. [13]). Letting $\lambda=k-(p a-2) /(r \alpha-1)$, we have $\lambda>1 /(r a-1)$ and it follows from (3.4) that if $E\left|X_{1}\right|^{r} \geq 1$, then 
$\sum_{n^{r a-1}>E\left|X_{1}\right|^{r}} n^{p a-2} P^{k}\left[\bar{S}_{n} \geq n^{a} /(2 k)\right] \leq \xi_{p, a, r}\left(E\left|X_{1}\right|^{r}\right)^{k} \sum_{n^{r a-1}>E\left|X_{1}\right|^{r}} n^{-\lambda(r a-1)}$

$$
\begin{aligned}
& \leq \zeta_{p, a, r}\left(E\left|X_{1}\right|^{r}\right)^{k-\lambda+(1 /(r a-1))} \\
& =\zeta_{p, a_{0} r}\left(E\left|X_{1}\right|^{r}\right)^{(p a-1) /(r a-1)}
\end{aligned}
$$

Also obviously

$$
\begin{aligned}
\sum_{n^{r a-1} \leq E\left|X_{1}\right|^{r}} n^{p a-2} P^{k}\left[\bar{S}_{n} \geq n^{a} /(2 k)\right] & \leq \sum_{n^{r a-1} \leq E\left|X_{1}\right|^{r}} n^{p a-2} \\
& \leq \eta_{p, a}\left(E\left|X_{1}\right|^{r(p a-1) /(r a-1)}\right.
\end{aligned}
$$

$$
\sum_{n=1}^{\infty} n^{p a-1} P\left[X_{1} \geq n^{\alpha} /(2 k)\right] \leq \theta_{p, a, r} E\left(X_{1}^{+}\right)^{p} \text {. }
$$

Using (3.2), (3.3), (3.5), (3.6) and (3.7), we then obtain the desired conclusion for the case $E\left|X_{1}\right|^{r} \geq 1$.

If $E\left|X_{1}\right|^{r}<1$, then it follows from (3.4) that

$$
\begin{aligned}
\sum_{n=1}^{\infty} n^{p a-2} p^{k}\left[\bar{S}_{n} \geq n^{\alpha} /(2 k)\right] & \leq \gamma_{p, a_{0} r}\left(E\left|X_{1}\right|^{r}\right)^{k} \sum_{n=1}^{\infty} n^{-\lambda(r a-1)} \\
& \leq \rho_{p, a_{, r}}\left(E\left|X_{1}\right|^{r}\right)^{(p a-1) /(r a-1)}
\end{aligned}
$$

Hence the desired conclusion also follows in this case.

Corollary. Suppose $X_{1}, X_{2}, \ldots$ are $i_{.} i_{.} d_{.}, E X_{1}=0$ and $E\left|X_{1}\right|^{r}<\infty$ for some $1 \leq r \leq 2$. Let $\alpha>1 / r$ and $p>1 / \alpha$. Then (1.7) is equivalent to each of the following statements:

$$
\begin{gathered}
E\left(X_{1}^{+}\right)^{p}<\infty \\
\sum n^{p a-2} P\left[\sup _{k \geq n} k^{-a} S_{k} \geq \epsilon\right]<\infty \text { for all } \epsilon>0 . \\
\sum n^{p a-2} P\left[S_{n} \geq \epsilon n^{a}\right]<\infty \text { for some } \epsilon>0 .
\end{gathered}
$$

Proof. Replacing $X_{i}$ by $X_{i} / \epsilon$ in Theorem 1 , it is easy to see from Theorem 1 that (3.9) $\Rightarrow$ (1.7). By Lemma 2 in $\$ 5$ below, (1.7) $\Rightarrow(3.10)$. Obviously $(3.10) \Rightarrow(3.11)$. By an argument due to Erdös [5] (see also Lemma 3 below) we can prove that $(3.11) \Rightarrow(3.9)$. 
The results in the above corollary have been partly established in [2] by different methods. In [2], the case $\alpha=1 / r$ and $p \geq r$ for $1 \leq r<2$ have also been considered and it is proved that in this case, (3.9) still implies (1.7). The following one-sided theorem on the convergence rate of tail probabilities deals with the case $a>1$. In this case, when $\alpha p>1$, it follows immediately from Theorem 3 of $[1]$ and the fact that $S_{n} \leq X_{1}^{+}+\cdots+X_{n}^{+}$, while the situation $\alpha p=1$ can be proved by using Theorem 1(iii) and Lemma 3 of [2].

Theorem 2. Suppose $X_{1}, X_{2}, \ldots$ are i.i.d., $\alpha>1$ and $\alpha p \geq 1$. If $E\left(X_{1}^{+}\right)^{p}<\infty$, then (1.7) holds, and consequently (3.10) also bolds when $a p>1$.

We remark that in Theorem 2, the relation (1.7) does not necessarily imply $E\left(X_{1}^{+}\right)^{p}<\infty$. To give an example, let $0<p<1, \alpha>1 / p, \gamma>p \alpha^{2} /(p \alpha-1)$. Setting $q=p a /\{\gamma(p \alpha-1)\}$, we have $0<q<p$. Choose $0<\nu<1$ such that $\gamma<\{\nu(p a-1)\}^{-1}$. Let $X_{1}, X_{2}, \ldots$ be i.i.d. random variables such that $E\left(X_{1}^{\dagger}\right)^{q}$ $<\infty, E\left(X_{1}^{+}\right)^{p}=\infty$ and $X_{1}^{-}$has the stable distribution with exponent $\nu$, i.e., the Laplace transform of $X_{1}^{-}$is given by $E \exp \left(-\lambda X_{1}^{-}\right)=\exp \left(-\lambda^{\nu}\right), \lambda>0$. Since $E\left(X_{1}^{\dagger}\right)^{q}<\infty$, it follows from the Marcinkiewicz-Zygmund strong law of large numbers that

$$
\lim _{n \rightarrow \infty} n^{-1 / a} \max _{j \leq n}\left(X_{1}^{+}+\cdots+X_{j}^{+}\right)=0 \text { a.e. }
$$

In particular, (3.12) holds along the subsequence $\left[m^{p a /(p a-1)}+m^{1 /(p a-1)}\right]$, and so

(3.13) $\lim _{m \rightarrow \infty} m^{-\gamma} \max _{j \leq m^{p a /(p a-1)}+m^{1 /(p a-1)}}\left(X_{1}^{+}+\cdots \cdot+X_{j}^{+}\right)=0$ a.e.

This in turn implies that

$$
\lim _{m \rightarrow \infty} m_{m}^{-\gamma /(+)}{ }_{p a /(p a-1)}{ }_{{ }^{\prime}}{ }^{1 /(p a-1)}=0 \text { a.e. }
$$

where we define $S_{r, t}^{(+)}=\Sigma_{r<j \leq[r]+t} X_{j}^{+}$, and $S_{r, t}^{(-)}=\Sigma_{r<j \leq[r]+t} X_{j}^{-}$. Now $j^{-1 / \nu} S_{r, j}^{(-)}$ has the same distribution as $X_{1}^{-}$, and it is well known that $P\left[X_{1}^{-} \leq t\right]=$ $o\left(\exp \left(-t^{-\nu}\right)\right)$ as $t \mid 0$. Since $\gamma<\{\nu(p \alpha-1)\}^{-1}$, it follows from the BorelCantelli lemma that

$$
\lim _{m \rightarrow \infty} m_{m^{p a /(p a-1)}, m^{1 /(p a-1)}}=\infty \text { a.e. }
$$

From (3.14) and (3.15), we obtain

$$
\lim _{m \rightarrow \infty} m^{-\gamma / S}{ }_{m a /(p a-1)}, m^{1 /(p a-1)}=-\infty \text { a.e. }
$$

Since $a /(p a-1)<\gamma,(3.16)$ implies that

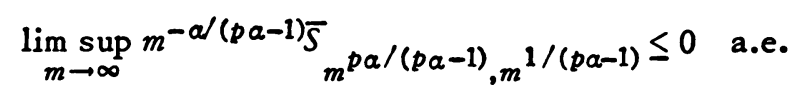


By Lemma 3 of [2], (3.17) is equivalent to (1.7). We remark that the above example is similar to the one given by Baum [18] in another context.

Thus we have seen that in Theorem 2, (1.7) does not necessarily imply $E\left(X_{1}^{+}\right)^{p}<\infty$. A sufficient condition which would guarantee this implication is $E\left|X_{1}\right|^{1 / \alpha}<\infty$. Under this additional condition, $\lim _{n \rightarrow \infty} n P\left[X_{1} \geq \epsilon n^{\alpha}\right]=0$, and by the Marcinkiewicz-Zygmund strong law of large numbers, $\lim _{n \rightarrow \infty} P\left[S_{n} \geq \epsilon n^{\alpha}\right]$ $=0$. Hence it can then be shown by the Erdös method that, in this case, (1.7) implies $E\left(X_{1}^{+}\right)^{p}<\infty$.

4. One-sided limit theorems for delayed sums and their relation to the convergence rate of tail probabilities. The quantity $S_{r, t}$ defined in (3.1) is called a delayed sum for the sequence $X_{n}$ (cf. [17, Vol. 1, p. 80]). In [2], the following strong law for delayed sums has been proved: If $X_{1}, X_{2}, \ldots$ are i.i.d. with $E X_{1}=0$ and $E\left|X_{1}\right|^{p}<\infty$ for some $p \geq 1$, then for every $0<\beta<$ $\min (1,2 / p)$,

$$
\lim _{n \rightarrow \infty} n^{-1 / p} \max _{1 \leq j \leq n}\left|S_{n, j}\right|=0 \text { a.e. }
$$

The corresponding one-sided limit theorem has also been obtained: If $E\left|X_{1}\right|^{r}$ $<\infty$ for some $1 \leq r \leq 2, E\left(X_{1}^{+}\right)^{p}<\infty$ for some $p \geq r$ and $E X_{1}=0$, then for every $0<\beta<r / p$ (or for every $0<\beta \leq 1 / p$ in the case $r=1$ ),

$$
\limsup _{n \rightarrow \infty} n^{-1 / p} \bar{S}_{n, n} \beta \leq 0 \text { a.e. }
$$

For $\alpha_{r}>1$, obviously (4.2) implies (3.17) which is in turn equivalent to (1.7). Thus based on the equivalence between (1.7) and (3.17) which holds for any $a>0$ and $a p>1$, we can prove theorems concerning the convergence rate of tail probabilities from the corresponding limit theorems for delayed. sums.

We note that while $\beta$ ranges from 0 to $\min (1,2 / p)$ in (4.1), the range of $\beta$ in (4.2) is from 0 to $r / p$. Our example in $\$ 2$ shows that we cannot extend the range of $\beta$ in (4.2). In that example, $r=1$ and we can take any $p>1$ since $X_{1}^{+}$is bounded. Now for any $0<\beta<1$, since $S_{n, n}$ has the same distribution as $S_{n} \beta$, it is easy to see from (2.4) that

$$
\underset{n \rightarrow \infty}{\limsup }(\delta / a) S_{n, n^{\beta}}(\beta \log n)^{\delta / n^{\beta}} \geq 1 \text { a.e. }
$$

Therefore if $\beta>1 / p$, then lim sup $\operatorname{sum}_{n \rightarrow \infty} n^{-1 / p} S_{n, n} \beta=\infty$ a.e. This shows that we cannot extend the range of $\beta$ in (4.2) beyond $r / p$. It is interesting to note that in spite of (2.4), we have for any $0<\beta<1$,

$$
\liminf _{n \rightarrow \infty} S_{n, n}(\log n)^{\delta} / n^{\beta}=-\infty \quad \text { a.e. }
$$


To prove (4.4), let $\left.Z_{n}=\left(X_{n+1}+\cdots+X_{n+[n} \beta\right]\right)(\log n)^{\delta} / n^{\beta}, Y_{n}=X_{n}(\log n)^{\delta} / n^{\beta}$. Suppose (4.4) is not true. Then by the zero-one law, there exists a constant $c$ such that $\lim \inf _{n \rightarrow \infty}\left(Y_{n}+Z_{n}\right) \geq c$ a.e. Since $Z_{n}$ is independent of $\left(Y_{1}, \ldots, Y_{n}\right)$ and $Z_{n} \stackrel{P}{\longrightarrow} a / \delta$, it follows from Lemma 1 in [11] that $\liminf _{n \rightarrow \infty} Y_{n} \geq c-(a / \delta)$ a.e. But $\lim _{n \rightarrow \infty} Y_{n}=-\infty$ since $E X_{1}\left(\log \left|X_{1}\right|\right)^{\delta}$ $=-\infty$, and so we have a contradiction.

The proof of the equivalence of (1.4), (1.5) and (1.6) in [11] is based on the following analogue of the law of the iterated logarithm for delayed sums: If $X_{1}, X_{2}, \ldots$ are i.i.d. and $0<\beta<1$, then

$$
E X_{1}=0, \quad E X_{1}^{2}=\sigma^{2} \text { and } E\left|X_{1}\right|^{2 / \beta}\left(\log ^{+}\left|X_{1}\right|+1\right)^{-1 / \beta}<\infty
$$

$$
\Leftrightarrow \limsup _{n \rightarrow \infty} \max _{1 \leq j \leq n}\left|S_{n, j}\right| /\left\{2(1-\beta) n^{\beta} \log n\right\}^{1 / 2}=\sigma \text { a.e. }
$$

Theorem 3 below gives the one-sided analogue of (4.5).

Theorem 3. Let $X_{1}, X_{2}, \ldots$ be i.i.d. random variables such that $E X_{1}=0$, $E X_{1}^{2}=\sigma^{2}(<\infty)$. Then for $0<\beta<1$, the following statements are equivalent:

$$
\begin{gathered}
\int_{\left[x_{1}>e\right]} X_{1}^{2 / \beta}\left(\log X_{1}\right)^{-1 / \beta} d P<\infty, \\
\underset{n \rightarrow \infty}{\limsup \bar{S}_{n, n} \beta} /\left\{2(1-\beta) n^{\beta} \log n\right\}^{1 / 2} \leq \sigma \quad \text { a.e., } \\
\underset{n \rightarrow \infty}{\limsup S_{n, n} \beta} /\left\{2(1-\beta) n^{\beta} \log n\right\}^{1 / 2}<\infty \text { a.e. }
\end{gathered}
$$

Lemma 1. Let $X$ be a nonnegative random variable such that $E g(X)<\infty$ for some nonnegative Borel function $g$. Then there exists a positive nondecreasing function $\psi$ on $[0, \infty)$ such that $\lim _{t \rightarrow \infty} \psi(t)=\infty, \lim _{t \rightarrow \infty} \psi\left(t^{p}\right) / \psi(t)$ $=1$ for all $p>0$ and $E \psi(X) g(X)<\infty$.

Proof. Let $F$ be the distribution function of $X$. Let $\left(n_{k}\right)_{k \geq 1}$ be a sequence of integers such that $n_{1} \geq 2, n_{k+1}>2^{n_{k}}$ and $\int\left[n_{k}, \infty\right) g(t) d F(t)<2^{-k}$. Let $n_{0}=0$ and define $\psi(t)=k^{1 / 2}$ for $n_{k-1} \leq t<n_{k}$. Obviously $\psi(t) \uparrow \infty$ as $t \uparrow \infty$ and

$$
\begin{aligned}
\int_{0}^{\infty} \psi(t) g(t) d F(t) & =\sum_{k=1}^{\infty} \int_{\left[n_{k-1}, n_{k}\right)} \psi(t) g(t) d F(t) \\
& \leq \int_{0}^{n} g(t) d F(t)+\sum_{k=2}^{\infty} k^{1 / 2} 2^{-(k-1)}<\infty .
\end{aligned}
$$


Proof of Theorem 3. First suppose that (4.6) holds. To prove (4.7), we shall use the idea of Hartman and Wintner [7] in truncating the random variables from below. By Lemma 1, we can choose a positive nondecreasing function $\psi$ on $[0, \infty)$ such that $\lim _{t \rightarrow \infty} \psi(t)=\infty, \lim _{t \rightarrow \infty} \psi\left(t^{p}\right) / \psi(t)=1$ for all $p>0$ and $E X_{1}^{2} \psi\left(\left|X_{1}\right|\right)<\infty$. Without loss of generality, we can assume that $\sigma>0$. Given $\delta>0$, we pick an integer $k>1$ such that $k-\beta k>1$ and then choose $\epsilon>0$ such that $\epsilon k<\delta$. Define

$$
\begin{aligned}
& X_{n}^{(1)}=X_{n} I_{\left[-n^{\beta / 2}\left(\log _{n}\right)^{-1 / 2}(\psi(n))^{-1 / 2} \leq X_{n} \leq n^{\beta / 2} /(\log n)\right]}, \\
& \left.X_{n}^{(2)}=X_{n} I_{\left[X_{n}^{<-n}\right.}^{\beta / 2}(\log n)^{-1 / 2}(\psi(n))^{-1 / 2}\right]^{\prime} \\
& X_{n}^{(3)}=X_{n} I_{[n}^{\left.\beta / 2 /(\log n)<X_{n}<\epsilon n^{\beta / 2}(\log n)^{1 / 2}\right]^{\prime}} \\
& \left.X_{n}^{(4)}=X_{n} I_{\left[X_{n} \geq \epsilon n\right.}^{\beta / 2}(\log n)^{1 / 2}\right]^{\circ}
\end{aligned}
$$

Let $U_{n}=X_{n}^{(1)}-E X_{n}^{(1)}$. Then $E U_{n}=0, E U_{n}^{2}=\sigma_{n}^{2} \rightarrow \sigma^{2}$ and

$$
\left|U_{n}\right| \leq 2 n^{\beta / 2}\left\{(\log n)^{-1}+(\log n)^{-1 / 2}(\psi(n))^{-1 / 2}\right\}=\gamma_{n}=o\left(n^{\beta / 2}(\log n)^{-1 / 2}\right) \text {. }
$$

Hence for $|t| \gamma_{j} \leq 1$,

$$
\exp \left\{t^{2} \sigma_{j}^{2}\left(1-|t| \gamma_{j}\right) / 2\right\} \leq E \exp \left(t U_{j}\right) \leq \exp \left\{t^{2} \sigma_{j}^{2}\left(1+1 / 2|t| \gamma_{j}\right) / 2\right\}
$$

(cf. [12, p. 255]). Therefore by Theorem 1 of [11],

$$
\text { (4.9) } \lim _{n \rightarrow \infty} \max _{1 \leq j \leq n}\left|U_{n+1}+\cdots+U_{n+j}\right| /\left\{2(1-\beta) n^{\beta} \log n\right\}^{1 / 2}=\sigma \text { a.e. }
$$

We note that, since $E X_{1}=0$, we have for all large $n$,

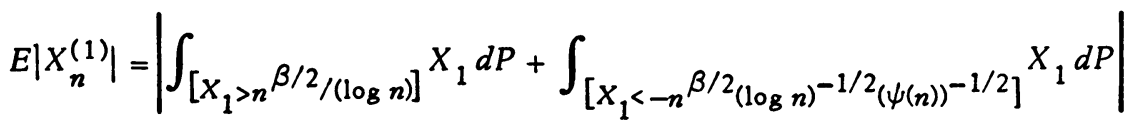

$$
\begin{aligned}
& \leq 2\left\{(2 / \beta)^{1 / \beta} n^{\beta / 2-1}(\log n)^{3 / \beta-1} \int_{\left[x_{1}>e\right]} X_{1}^{2 / \beta}\left(\log X_{1}\right)^{-1 / \beta} d P\right. \\
& \left.+n^{-\beta / 2}(\log n)^{1 / 2}(\psi(n))^{-1 / 2} E X_{1}^{2} \psi\left(\left|X_{1}\right|\right)\right\} \text {. }
\end{aligned}
$$

Therefore

$$
\lim _{n \rightarrow \infty} \sum_{j=1}^{[n} E\left|X_{n+j}^{(1)}\right| /\left\{n^{\beta} \log n\right\}^{1 / 2}=0
$$


It is obvious that

(4.11) $\quad \limsup _{n \rightarrow \infty} \max _{1 \leq j \leq n}\left(X_{n+1}^{(2)}+\cdots+X_{n+j}^{(2)}\right) /\left\{n^{\beta} \log n\right\}^{1 / 2} \leq 0 \quad$ a.e.;

$$
\lim _{n \rightarrow \infty} \max _{1 \leq j \leq n} \beta\left(X_{n+1}^{(4)}+\cdots+X_{n+j}^{(4)}\right) /\left\{n^{\beta} \log n\right\}^{1 / 2}=0 \text { a.e. }
$$

Since $0 \leq X_{n}^{(3)}<\epsilon n^{\beta / 2}(\log n)^{1 / 2}$, it can be shown as in the proof of Theorem 2 in [11] that

$$
\begin{aligned}
P\left[\max _{1 \leq j \leq n}\left(X_{n+1}^{(3)}+\cdots+X_{n+j}^{(3)}\right)\right. & \left.\geq \delta\left(n^{\beta} \log n\right)^{1 / 2}\right] \\
& \leq P\left[\sum_{j=1}^{\left[n^{\beta} 1\right.} X_{n+j}^{(3)} /\left\{\epsilon n^{\beta / 2}(\log n)^{1 / 2}\right\} \geq k\right] \\
& =O\left(n^{\beta k}\left\{(\log n)^{3 / \beta} / n\right\}^{k}\right) .
\end{aligned}
$$

Since $k-\beta k>1$, an application of the Borel-Cantelli lemma gives (4.13) $\limsup _{n \rightarrow \infty} \max _{1 \leq j \leq n}\left(X_{n+1}^{(3)}+\cdots+X_{n+j}^{(3)}\right) /\left\{n^{\beta} \log n\right\}^{1 / 2} \leq \delta$ a.e. As $\delta$ is: arbitrary, we obtain (4.7) from (4.9), (4.10), (4.11), (4.12) and (4.13).

Obviously (4.7) implies (4.8). Now assume (4.8). By the zero-one law, there exists a finite constant $c$ such that the lim sup in (4.8) is $\leq c$ a.e. Define

$$
\begin{aligned}
& Y_{n}=X_{n} /\left\{2(1-\beta) n^{\beta} \log n\right\}^{1 / 2}, \\
& Z_{n}=\left(X_{n+1}+\cdots+X_{n+\left[n^{\beta}\right]}\right) /\left\{2(1-\beta) n^{\beta} \log n\right\}^{1 / 2} .
\end{aligned}
$$

Since $E X_{1}=0$ and $E X_{1}^{2}=\sigma^{2}, Z_{n} \stackrel{P}{\longrightarrow} 0$. Obviously $Z_{n}$ is independent of $\left(Y_{1}, \ldots, Y_{n}\right)$. Since $\lim \sup _{n \rightarrow \infty}\left(Y_{n}+Z_{n}\right) \leq c$ a.e., we obtain using Lemma 1 of [11] that $\lim \sup _{n \rightarrow \infty} Y_{n} \leq c$ a.e. From this, (4.6) follows easily.

By using a similar argument as the proof in [11] of the equivalence of (1.4), (1.5) and (1.6), we can obtain from Theorem 3 the following one-sided theorem on the tail distribution of sample sums.

Theorem 4. Suppose $X_{1}, X_{2}, \ldots$ are i.i.d., $E X_{1}=0, E X_{1}^{2}=\sigma^{2}(<\infty)$. Then for any $p>2$, the following statements are equivalent:

$$
\int_{\left[X_{1}>e\right]} X_{1}^{p}\left(\log X_{1}\right)^{-p / 2} d P<\infty,
$$

$$
\sum n^{p / 2-2} P\left[\bar{S}_{n} \geq \epsilon(n \log n)^{1 / 2}\right]<\infty \quad \text { for all } \epsilon>\sigma(p-2)^{1 / 2} \text {, }
$$


(4.16) $\sum n^{p / 2-2} P\left[\sup _{k \geq n}(k \log k)^{-1 / 2} S_{k} \geq \epsilon\right]<\infty$ for all $\epsilon>\sigma(p-2)^{1 / 2}$,

$$
\sum n^{p / 2-2} P\left[S_{n} \geq \epsilon(n \log n)^{1 / 2}\right]<\infty \text { for some } \epsilon>0 \text {. }
$$

5. Applications to the moments of the largest excess and the last time of boundary crossings. In this section, we shall consider moments of the last time $T(\epsilon, \alpha)$ and of the largest excess $M(\epsilon, \alpha)$ of certain boundary crossings for the sample sums $S_{n}$ as defined in $\S 1$. These are related to the moments of $T_{1}(\epsilon, \alpha), M_{1}(\epsilon, \alpha)$ for the original sample observations $X_{n}$. We now introduce the following notation: For $p>0, a>0$,

$$
J(\epsilon ; p, \alpha)=\int_{0}^{\infty} t^{p a-2} P\left[\bar{S}_{t} \geq \epsilon t^{\alpha}\right] d t,
$$

$I(\epsilon ; p, a)=\int_{0}^{\infty} t^{p a-2} P\left[\sup _{k \geq t} k^{-a} s_{k} \geq \epsilon\right] d t$,

$$
m(\epsilon ; p, \alpha)=E(M(\epsilon, \alpha))^{(p a-1) / \alpha}, \quad M(\epsilon, \alpha)=\sup _{n \geq 0}\left(S_{n}-\epsilon n^{\alpha}\right),
$$

(5.4) $\tau(\epsilon ; p, a)=E(T(\epsilon, a))^{p a-1}, \quad T(\epsilon, a)=\sup \left\{n \geq 1: S_{n} \geq \epsilon n^{a}\right\}(\sup \phi=0)$,

(5.5) $s(\epsilon ; p, \alpha)=E\left(\bar{S}_{T(\epsilon, \alpha)}\right)^{(p a-1) / \alpha}$,

(5.6) $J_{1}(\epsilon ; p, \alpha)=\int_{0}^{\infty} t^{p a-2} P\left[\bar{X}_{t} \geq \epsilon t^{\alpha}\right] d t$,

(5.7) $I_{1}(\epsilon ; p, \alpha)=\int_{0}^{\infty} t^{p a-2} P\left[\sup _{k \geq t} k^{-a} X_{k} \geq \epsilon\right] d t$,

(5.8) $m_{1}(\epsilon ; p, \alpha)=E\left(M_{1}(\epsilon, \alpha)\right)^{(p a-1) / \alpha}, \quad M_{1}(\epsilon, \alpha)=\sup _{n \geq 0}\left(X_{n}-\epsilon n^{\alpha}\right)$,

(5.9) $\tau_{1}(\epsilon ; p, \alpha)=E\left(T_{1}(\epsilon, \alpha)\right)^{p a-1}$, $T_{1}(\epsilon, a)=\sup \left\{n \geq 1: X_{n} \geq \epsilon n^{a}\right\}$,

$(5.10) s_{1}(\epsilon ; p, \alpha)=E\left(\bar{X}_{T_{1}(\epsilon, a)}\right)^{(p a-1) / a}$.

Lemma 2. Let $S_{1}, S_{2}, \ldots$ be any sequence of random variables (not necessarily sample sums). Define $S_{0}=\bar{S}_{0}=0, \bar{S}_{t}=\bar{S}_{[t]}=\max _{1 \leq j \leq[t]} S_{j}$, and define $J(\epsilon ; p, \alpha), I(\epsilon ; p, \alpha), m(\epsilon ; p, \alpha), \tau(\epsilon ; p, \alpha)$ and $s(\epsilon ; p, \alpha)$ as in (5.1)-(5.5). Then, for any positive constants $\epsilon, p, \alpha$ with $p a>1$,

$$
(p a-1) \epsilon^{(p a-1) / a} I(2 \epsilon ; p, \alpha) \leq m(\epsilon ; p, a)
$$

$$
\begin{aligned}
& \leq\left(2^{(p a-1) / \alpha}-1\right)^{-1}(p a-1) \epsilon^{(p a-1) / \alpha} J(\epsilon / 2 ; p, \alpha), \\
& \tau(\epsilon ; p, \alpha) \leq(p a-1) I(\epsilon ; p, \alpha),
\end{aligned}
$$


(5.13) $\epsilon^{(p a-1) / a} \tau(\epsilon ; p, \alpha) \leq s(\epsilon ; p, \alpha) \leq K_{p, a}\left\{\epsilon^{(p a-1) / a} \tau(\epsilon ; p, \alpha)+m(\epsilon ; p, \alpha)\right\}$, where $K_{p, a}=1$ if $p a-1 \leq \alpha$ and $K_{p, a}=2^{((p a-1) / a)-1}$ if $p a-1>a$.

Proof. Let $r=(p \alpha-1) / \alpha$. We note that

$$
\begin{aligned}
\epsilon^{-r} m(\epsilon ; p, \alpha) & =\int_{0}^{\infty} P\left[\sup _{n \geq 1}\left(S_{n}-\epsilon n^{\alpha}\right) \geq \epsilon t^{1 / r}\right] d t \\
& \leq \sum_{k=1}^{\infty} \int_{0}^{\infty} P\left[\underset{\left(2^{k-1}-1\right) t^{1 / r} n^{\alpha} \leq\left(2^{k}-1\right) t^{1 / r}}{\left.\left.\operatorname{su}_{n}-\epsilon n^{\alpha}\right) \geq \epsilon t^{1 / r}\right] d t}\right. \\
& \leq \sum_{k=1}^{\infty} \int_{0}^{\infty} P\left[\bar{S}_{\left(2^{k} t^{1 / r}\right)^{1 / a} \geq 2^{k-1} \epsilon t}{ }^{1 / r}\right] d t \\
& =\sum_{k=1}^{\infty} 2^{-k r}(p a-1) \int_{0}^{\infty} u^{p a-2} P\left[\bar{S}_{u} \geq(\epsilon / 2) u^{a}\right] d u .
\end{aligned}
$$

To complete the proof of (5.11), we have

$$
\begin{aligned}
I(2 \epsilon ; p, \alpha) & =\int_{0}^{\infty} t^{p a-2} P\left[\sup _{k \geq t} k^{-a} S_{k} \geq 2 \epsilon\right] d t \\
& \leq \int_{0}^{\infty} t^{p a-2} P\left[\sup _{n \geq 0}\left(S_{n}-\epsilon n^{\alpha}\right) \geq \epsilon t^{a}\right] d t=(p a-1)^{-1} E(M(\epsilon, \alpha) / \epsilon)^{r} .
\end{aligned}
$$

Since $P[T(\epsilon, a) \geq t] \leq P\left[\sup _{k \geq t} k^{-a} S_{k} \geq \epsilon\right]$, it is easy to see (5.12). Finally, (5.13) follows immediately from the fact that

$$
\epsilon T^{a}(\epsilon, \alpha) \leq \bar{S}_{T(\epsilon, a)} \leq \epsilon T^{a}(\epsilon, a)+\sup _{n \geq 0}\left(S_{n}-\epsilon n^{a}\right) .
$$

We remark that if $X_{1}, X_{2}, \ldots$ are i.i.d. random variables with $E X_{1}=0$ and $\left(S_{n}\right)$ is the sequence of partial sums, then Lemma 2 and inequality (1.9) imply that for $\alpha>1 / 2, p>1 / 2$ and $\epsilon>0$,

$$
m(\epsilon ; p, \alpha) \leq A_{p, a} \epsilon^{(p a-1) / a}\left\{E\left(X^{+} / \epsilon\right)^{p}+\left(E\left(X_{1} / \epsilon\right)^{2}\right)^{(p a-1) /(2 a-1)}\right\}
$$

where $A_{p, \alpha}>0$ is a universal constant depending only on $p$ and $\alpha$. In the case $p=2$ and $\alpha=1$, this reduces to $E\left(\sup _{n \geq 0}\left(S_{n}-\epsilon n\right)\right) \leq A \epsilon^{-1} E X_{1}^{2}$, a result obtained by Kingman [10] by a different approach. In fact, Kingman showed that the constant $A$ in the above upper bound can be taken to be $1 / 2$. This is a very sharp bound for small $\epsilon$ in view of the fact that

$$
\lim _{\epsilon 10} \epsilon E\left(\sup _{n \geq 0}\left(S_{n}-\epsilon n\right)\right)=1 / 2 E X_{1}^{2}
$$

(see Theorem 7 below). 
Lemma 3. Suppose $a>1 / 2$ and $X_{1}, X_{2}, \ldots$ are i.i.d. random variables with $E\left|X_{1}\right|^{1 / a}<\infty$. Assume further that $E X_{1}=0$ in the case $\alpha \leq 1$. Letting $S_{n}=X_{1}+\cdots+X_{n}$, we have for any $\gamma>0, \epsilon>0$,

$$
E(T(\epsilon, a))^{\gamma}<\infty \Rightarrow E\left(T_{1}(2 \epsilon, a)\right)^{\gamma}<\infty .
$$

Proof. Set $A_{k}=\left[X_{k} \geq 2 \epsilon k^{\alpha}\right], B_{k}=\left[\left|S_{k-1}\right| \leq \epsilon k^{\alpha}\right]$. By the MarcinkiewiczZygmund strong law of large numbers, $n^{-a} S_{n} \rightarrow 0$ a.e., and so $\lim _{k \rightarrow \infty} P\left(B_{k}\right.$ $=1$. Since $E\left|X_{1}\right|^{1 / a}<\infty, \lim _{m \rightarrow \infty} P\left(\bigcup_{j=m}^{\infty} A_{j}\right)=P\left(A_{n}\right.$ i.o. $)=0$, and so we can choose $m_{0}$ such that $P\left(B_{k}\right)-P\left(\bigcup_{j=m}^{\infty} A_{j}\right) \geq 1 / 2$ if $k \geq m \geq m_{0}$. By an argument due to Erdös [5], we then obtain that, for $m \geq m_{0}$,

$$
\begin{aligned}
P[T(\epsilon, \alpha) \geq m] & \geq \sum_{k=m}^{\infty}\left\{P\left(A_{k} \cap B_{k}\right)-P\left(A_{k} \cap\left(\bigcup_{j=m}^{k-1} A_{j}\right)\right)\right\} \\
& \geq \frac{1}{2} \sum_{k=m}^{\infty} P\left(A_{k}\right) \geq \frac{1}{2} P\left[T_{1}(2 \epsilon, \alpha) \geq m\right] .
\end{aligned}
$$

The desired conclusion then follows.

Lemma 4. Suppose $X_{1}, X_{2}, \ldots$ are i.i.d. random variables and $a>0$, $p>1 / a$. Then for all $\epsilon>0$,

$$
(p a-1) J_{1}(\epsilon ; p, \alpha) \leq \tau_{1}\left(2^{-a} \epsilon ; p, \alpha\right) \text { and } p a J_{1}(\epsilon ; p, \alpha) \leq \epsilon^{-p} E\left(X_{1}^{+}\right) p \text {. }
$$

Furthermore, if $J_{1}(\epsilon ; p, \alpha)<\infty$ for some $\epsilon>0$, then $E\left(X_{1}^{+}\right)^{p}<\infty$ and

$$
J_{1}(1 ; p, \alpha) \geq\left(E\left(X_{1}^{+}\right)^{p}-1\right) /\left\{2 p a\left(1+E\left(X_{1}^{+}\right)^{1 / \alpha}\right)\right\} .
$$

Proof. We note that

$$
\begin{aligned}
\tau_{1}\left(2^{-a} \epsilon ; p, \alpha\right) & =(p a-1) \int_{0}^{\infty} t^{p a-2} P\left[T_{1}\left(2^{-a} \epsilon, \alpha\right) \geq t\right] d t \\
& =(p \alpha-1) \int_{0}^{\infty} t^{p a-2} P\left[X_{n} \geq 2^{-a} \epsilon n^{\alpha} \text { for some } n \geq t\right] d t \\
& \geq(p \alpha-1) \int_{0}^{\infty} t^{p a-2} P\left[\max _{t<n \leq 2 t} X_{n} \geq \epsilon t^{\alpha}\right] d t \\
& \geq(p \alpha-1) \int_{0}^{\infty} t^{p a-2} P\left[\bar{X}_{t} \geq \epsilon t^{\alpha}\right] d t .
\end{aligned}
$$

It is also easy to see that

$$
\begin{aligned}
p \alpha J_{1}(\epsilon ; p, \alpha) & =p \alpha \int_{1}^{\infty} t^{p a-2} P\left[\bar{X}_{t} \geq \epsilon t^{\alpha}\right] d t \\
& \leq p \alpha \int_{1}^{\infty} t^{p a-1} P\left[X_{1} \geq \epsilon^{\alpha}\right] d t \leq \epsilon^{-p} E\left(X_{1}^{+}\right)^{p} .
\end{aligned}
$$


To prove (5.16), for any $c>0$, define $X_{i}(c)=X_{i} I_{\left[X_{i} \leq c\right]}, \bar{X}_{t}(c)=$ $\max _{1 \leq i \leq t} X_{i}(c), \bar{X}_{0}(c)=X_{0}(c)=0$. Put $n=[t]$, and note that for $t \geq 1$,

$$
\begin{aligned}
P\left[\bar{X}_{t}(c) \geq t^{a}\right]= & P\left[X_{1}(c) \geq t^{a}\right]+P\left[X_{2}(c) \geq t^{a}\right] P\left[X_{1}(c)<t^{a}\right] \\
& +\cdots+P\left[X_{n}(c) \geq t^{a}\right] P\left[X_{n-1}(c)<t^{a}\right] \ldots P\left[X_{1}(c)<t^{a}\right] \\
\geq & n P\left[X_{1}(c) \geq t^{a}\right] P^{n}\left[X_{1}(c)<t^{a}\right] \\
= & n P\left[X_{1}(c) \geq t^{a}\right]\left(1-P\left[\bar{X}_{t} \geq t^{a}\right]\right) .
\end{aligned}
$$

Therefore, for $t \geq 1$,

$1 / 2 t P\left[X_{1}(c) \geq t^{a}\right] \leq n P\left[X_{1}(c) \geq t^{\alpha}\right] \leq P\left[\bar{X}_{t}(c) \geq t^{a}\right]\left\{1+n P\left[X_{1}(c) \geq t^{a}\right]\right\}$

$$
\leq P\left[\bar{X}_{t}(c) \geq t^{a}\right]\left\{1+E\left(X_{1}^{+}(c)\right)^{1 / a}\right\}, \quad \text { by the Markov inequality. }
$$

From this, it follows that

$$
E\left(X_{1}^{+}(c)\right)^{p}-1 \leq p a \int_{1}^{\infty} t^{p a-1} P\left[X_{1}(c) \geq t^{a}\right] d t
$$

$$
\leq 2 p \alpha\left\{1+E\left(X_{1}^{+}(c)\right)^{1 / a}\right\} \int_{1}^{\infty} t^{p a-2} P\left[\bar{X}_{t}(c) \geq t^{a}\right] d t \text {. }
$$

Suppose $E\left(X_{1}^{+}\right)^{p}=\infty$. Then $E\left(X_{1}^{+}(c)\right)^{1 / a}=o\left(E\left(X_{1}^{+}(c)\right)^{p}\right)$ as $c \rightarrow \infty$, and so it easily follows from $(5.17)$ that $J_{1}(1 ; p, a)=\infty$. Hence $J_{1}(1 ; p, a)<\infty$ implies that $E\left(X_{1}^{+}\right)^{p}<\infty$, and in this case, letting $c \uparrow \infty$ in (5.17), we obtain (5.16).

Lemmas 2,3 and 4 together with the results in $\$ 3$ give the following theorem.

Theorem 5. Suppose $X_{1}, X_{2}, \ldots$ are i.i.d. random variables, $S_{n}=X_{1}+$ $\cdots+X_{n}$, and $\alpha>0, p>0$ such that $\alpha p>1$.

(i) If $E\left(X_{1}^{+}\right)^{p}<\infty$, then for every $\epsilon>0, I_{1}(\epsilon ; p, \alpha), J_{1}(\epsilon ; p, \alpha)$, $m_{1}(\epsilon ; p, \alpha), \tau_{1}(\epsilon ; p, \alpha)$ and $s_{1}(\epsilon ; p, \alpha)$ are all finite. Conversely, if one of the above five quantities is finite for some $\epsilon>0$, then $E\left(X_{1}^{+}\right)^{p}<\infty$.

(ii) Suppose $E\left(X_{1}^{+}\right)^{p}<\infty$ and $a>1 / 2$. In the case $a=1$, assume further that $E X_{1}=0$. In the case $a<1$, assume further that $E X_{1}=0$ and $E\left|X_{1}\right|^{r}<\infty$ for some $2 \geq r>1 / a$. Under these assumptions, $I(\epsilon ; p, \alpha)$, $J(\epsilon ; p, \alpha), m(\epsilon ; p, \alpha), \tau(\epsilon ; p, \alpha)$ and $s(\epsilon ; p, \alpha)$ are finite for all $\epsilon>0$.

(iii) Suppose $a>1 / 2$ and $E\left|X_{1}\right|^{1 / \alpha}<\infty$. Assume further that $E X_{1}=0$ in the case $\alpha \leq 1$. If one of $J(\epsilon ; p, \alpha), J(\epsilon ; p, \alpha), m(\epsilon ; p, \alpha), \tau(\epsilon ; p, \alpha)$ and $s(\epsilon ; p, \alpha)$ is finite for some $\epsilon>0$, then $E\left(X_{1}^{+}\right)^{p}<\infty$. 
6. The limiting distribution and limiting moments of the last time and largest excess of boundary crossings for sample sums. The following theorem gives the limiting distribution of $T(\epsilon, \alpha), M(\epsilon, a)$ and $S_{T(\epsilon, a)}$ as $\epsilon \downarrow 0$.

Theorem 6. Suppose $W(t), t \geq 0$, is the standard Wiener process and $X_{1}$, $X_{2}, \ldots$ are i.i.d. random variables with $E X_{1}=0, E X_{1}^{2}=1$. Let $S_{n}=X_{1}+$ $\cdots+X_{n}, \alpha>1 / 2$, and define $M(\epsilon, \alpha)$ and $T(\epsilon, \alpha)$ for any $\epsilon>0$ by (5.3) and (5.4). Let $M^{*}(a)=\sup _{t \geq 0}\left(W(t)-t^{a}\right), T^{*}(a)=\sup \left\{t \geq 0: W(t) \geq t^{a}\right\}$. Then as $\in \downarrow 10$,

$$
\begin{aligned}
& \epsilon^{2 /(2 a-1)} T(\epsilon, \alpha) \stackrel{D}{\longrightarrow} T^{*}(\alpha), \\
& \epsilon^{1 /(2 a-1)} M(\epsilon, \alpha) \stackrel{D}{\longrightarrow} M^{*}(\alpha), \\
& \epsilon^{1 /(2 a-1)} S_{T(\epsilon, a)} \stackrel{D}{\longrightarrow}\left(T^{*}(a)\right)^{a} .
\end{aligned}
$$

Proof. (6.1) and (6.2) can be proved by an argument similar to that of Robbins, Siegmund and Wendel [15] or that of Müller, [19], who considered the problem in the case $a=1$. Alternatively we can use a result of Robbins and Siegmund [14, Theorem 2] in the following way. For any $x>0$,

$$
\begin{aligned}
P\left[\epsilon^{2 /(2 a-1)} T(\epsilon, a) \geq x\right] \\
\quad=P\left[S_{n} \geq \epsilon n^{a} \text { for some } n \geq \epsilon^{-2 /(2 a-1)} x\right] \\
=P\left[S_{n} \geq \sqrt{m}(n / m)^{a} \text { for some } n \geq x m\right], \text { where } m=\epsilon^{-2 /(2 a-1)}, \\
\quad \rightarrow P\left[W(t) \geq t^{a} \text { for some } t \geq x\right] \text { as } m \rightarrow \infty .
\end{aligned}
$$

Similarly, given any $x>0$, if we apply part (ii) of Theorem 2 in [14], where we set $g(t)=t^{a}+x$, then

$$
\begin{aligned}
& P\left[\epsilon^{1 /(2 a-1)} M(\epsilon, \alpha)>x\right]=P\left[m^{-1 / 2} \sup _{n \geq 1}\left(S_{n}-\sqrt{m}(n / m)^{a}\right)>x\right], \\
& \text { where } m=\epsilon^{-2 /(2 a-1)}, \\
&=P\left[m^{-1 / 2} S_{n}>(n / m)^{\alpha}+x \text { for some } n \geq 1\right] \\
& \rightarrow P\left[W(t)>t^{a}+x \text { for some } t>0\right] \text { as } m \rightarrow \infty .
\end{aligned}
$$

To prove (6.3), we note that $S_{T(\epsilon, a)} \leq \epsilon(T(\epsilon, a)+1)^{a}-X_{T(\epsilon, a)+1}$ and so

$$
\begin{aligned}
\left\{\epsilon^{2 /(2 a-1)}(T(\epsilon, a)+1)\right\}^{a}-\epsilon^{1 /(2 a-1)} X_{T(\epsilon, a)+1} \\
\geq \epsilon^{1 /(2 a-1)} S_{T(\epsilon, a)} \geq\left(\epsilon^{2 /(2 a-1)} T(\epsilon, a)\right)^{a} .
\end{aligned}
$$


Since $E X_{1}^{2}<\infty$ implies that $n^{-1 / 2} X_{n} \rightarrow 0$ a.e., it follows that

$$
(T(\epsilon, a))^{-1 / 2} X_{T(\epsilon, a)+1} \stackrel{P}{\longrightarrow} 0 .
$$

By (6.1), $\epsilon^{1 /(2 \alpha-1)}(T(\epsilon, \alpha))^{1 / 2} \stackrel{D}{\longrightarrow}\left(T^{*}(\alpha)\right)^{1 / 2}$. Therefore

$$
\epsilon^{1 /(2 a-1)} X_{T(\epsilon, a)+1} \stackrel{P}{\longrightarrow} 0 .
$$

From (6.1), (6.4) and (6.5), it is easy to see that (6.3) holds.

By making use of the inequality (1.9), we now obtain from Theorem 6 the limiting moments of $T(\epsilon, \alpha), M(\epsilon, \alpha)$ and $S_{T(\epsilon, \alpha)}$ as $\epsilon \downarrow 0$.

Theorem 7. With the same notations and assumptions as in Theorem 6 , if $E\left(X_{1}^{+}\right)^{p}<\infty$ for some $p>2$, then for any $a>1 / 2$,

$$
\begin{gathered}
\lim _{\epsilon \downarrow 0} \epsilon^{2(p a-1) /(2 a-1)} E(T(\epsilon, \alpha))^{p a-1}=E\left(T^{*}(\alpha)\right)^{p a-1}, \\
\lim _{\epsilon \downarrow 0} \epsilon^{(p a-1) /\{a(2 a-1)\}_{1}} E(M(\epsilon, \alpha))^{(p a-1) / a}=E\left(M^{*}(\alpha)\right)^{(p a-1) / a}, \\
\lim _{\epsilon \downarrow 0} \epsilon^{(p a-1) /\{a(2 a-1)\}} E\left(S_{T(\epsilon, a)}\right)^{(p a-1) / a}=E\left(T^{*}(a)\right)^{p a-1} .
\end{gathered}
$$

The above relations also hold for $2 \geq p>1 / a$.

Proof. In view of Theorem 6 , we need only show that $\left(\epsilon^{2 \prime^{\prime}(2 a-1)} T(\epsilon, a)\right)^{p a-1}$ and $\left(\epsilon^{1 /(2 a-1)} M(\epsilon, \alpha)\right)^{(p a-1) / a}, 0<\epsilon \leq 1$, are uniformly integrable, as this will in turn imply that $\left(\epsilon^{1 /(2 a-1)} S_{T(\epsilon, \alpha)}\right)^{(p a-1) / a}, 0<\epsilon \leq 1$, is also uniformly integrable by the inequality (5.14). First consider the case $p \geq 2$. Let $0<\delta$ $<1 / 2$ and define

$$
X_{i}^{\prime}=X_{i} I\left[\left|X_{i}\right| \leq K\right]-E X_{i} I\left[\left|X_{i}\right| \leq K\right], \quad X_{i}^{n}=X_{i} I_{[}\left[\left|X_{i}\right|>K\right]-E X_{i} I\left[\left|X_{i}\right|>K\right],
$$

where $K>0$ is so chosen that

$$
E\left(\left(X_{1}^{\prime \prime} / \delta\right)^{+}\right)^{p}+\left(E\left(X_{1}^{\prime \prime} / \delta\right)^{2}\right)^{(p a-1) /(2 a-1)} \leq \delta .
$$

Let

$$
S_{n}^{\prime}=X_{1}^{\prime}+\cdots+X_{n}^{\prime}, S_{n}^{\prime \prime}=X_{1}^{\prime \prime}+\cdots+X_{n}^{\prime \prime}
$$

and define $T^{\prime}(\epsilon, \alpha), M^{\prime}(\epsilon, a)$ for $S_{n}^{\prime}, T^{\prime \prime}(\epsilon, \alpha)$ and $M^{\prime \prime}(\epsilon, \alpha)$ for $S_{n}^{\prime \prime}$. Using Lemma 2 and (1.9), we obtain that for $0<\epsilon \leq 1$, 
(6.8)

$$
\begin{aligned}
& \epsilon^{2(p a-1) /(2 a-1)} E\left(T^{n}(\delta \epsilon, a)\right)^{p a-1}
\end{aligned}
$$

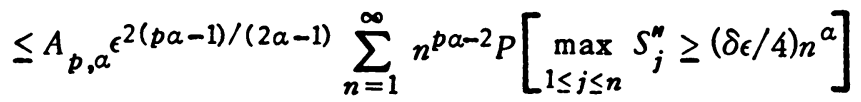

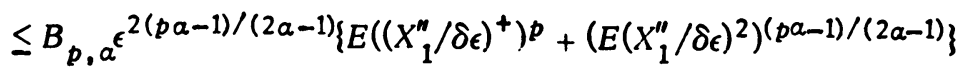

$$
\begin{aligned}
& \leq B_{p, a} \delta \text {, noting that } p \leq 2(p a-1) /(2 a-1) \text { since } p \geq 2 \text {. }
\end{aligned}
$$

The constants $A_{p, a}$ and $B_{p, a}$ above depend only on $p$ and $\alpha$. Now take any $q>p$ and by a similar argument as in (6.8), we have

$$
\begin{aligned}
& E\left(\epsilon^{2 /(2 a-1)} T^{\prime}((1-\delta) \epsilon, a)\right)^{q a-1} \\
& \leq C_{q, a}\left\{E\left(\left(X_{1}^{\prime}\right)^{+}\right)^{q}+\left(E\left(X_{1}^{\prime}\right)^{2}\right)^{(q a-1) /(2 a-1)}\right\},
\end{aligned}
$$

where $C_{q, a}$ is a positive constant depending only on $q$ and $\alpha$. Hence setting $Z(\epsilon, \delta)=\left(\epsilon^{2 /(2 a-1)} T^{\prime}((1-\delta) \epsilon, \alpha)\right)^{p a-1}$, we have the uniform integrability of $Z(\epsilon, \delta), 0<\epsilon \leq 1$, and so we can choose $\eta>0$ such that if $P(A)<\eta$, then $E Z(\epsilon, \delta) I_{A}<\delta$ for all $0<\epsilon \leq 1$. Since $T(\epsilon, \alpha) \leq T^{\prime}((1-\delta) \epsilon, \alpha)+T^{\prime \prime}(\delta \epsilon, \alpha)$, we have established the uniform integrability of $\left(\epsilon^{2 /(2 a-1)} T(\epsilon, \alpha)\right)^{p a-1}, 0<\epsilon \leq 1$, in the case $p \geq 2$.

Now let $2>p>1 / \alpha$. Then by what we just proved, $\left(\epsilon^{2 /(2 a-1)} T(\epsilon, \alpha)\right)^{2 a-1}$ is uniformly integrable, and so $\left(\epsilon^{2 /(2 a-1)} T(\epsilon, \alpha)\right)^{p a-1}$ is also uniformly integrable. The desired conclusion for $M(\epsilon, a)$ can be similarly proved.

Theorem 7 gives the asymptotic behavior of $\tau(\epsilon ; p, \alpha)$ and $m(\epsilon ; p, \alpha)$ as $\epsilon \downarrow 0$. It is also interesting to investigate the asymptotic behavior of $J(\epsilon ; p, \alpha)$ and $I(\epsilon ; p, \alpha)$. This is given in the following theorem.

Theorem 8. With the same notations and assumptions as in Theorem 6 , define $J(\epsilon ; p, a), I(\epsilon ; p, \alpha)$ by (5.1) and (5.2), and let $\Phi$ denote the distribution function of the standard normal distribution. If $E\left(X_{1}^{+}\right)^{p}<\infty$ for some $p \geq 2$, then for any $a>1 / 2$,

$$
\lim _{\epsilon \downarrow 0} \epsilon^{2(p a-1) /(2 a-1)} J(\epsilon ; p, \alpha)=2 \int_{0}^{\infty} t^{p a-2}\left(1-\Phi\left(t^{(2 a-1) / 2}\right)\right) d t
$$

$$
=2 \lim _{\epsilon \downarrow 0} \epsilon^{2(p a-1) /(2 a-1)} \int_{0}^{\infty} t^{p a-2} p\left[s_{t} \geq \epsilon t^{\alpha}\right] d t,
$$

$$
\lim _{\epsilon ! 0}(p a-1) \epsilon^{2(p a-1) /(2 a-1)} I(\epsilon ; p, \alpha)=E\left(T^{*}(\alpha)\right) p a-1 .
$$

Proof. To prove (6.10), we note that by a change of variable, we can write 
$J(\epsilon ; p, \alpha)$

(6.12)

$$
\begin{aligned}
& =\epsilon^{-2(p a-1) /(2 a-1)} \\
& \cdot \int_{0}^{\infty} u^{p a-2} P\left[\bar{S}_{u \epsilon-2 /(2 a-1)} \geq \epsilon^{-1 /(2 a-1)} u^{a}\right] d u .
\end{aligned}
$$

By Donsker's invariance principle, for any $u>0$,

$$
\begin{aligned}
\lim _{\epsilon \downarrow 0} P\left[\bar{S}_{u \epsilon^{-2 /(2 a-1)}} \geq \epsilon^{-1 /(2 a-1)} u^{a}\right] \\
\quad=P\left[\max _{0 \leq t \leq 1} W(t) \geq u^{a-1 / 2}\right]=2\left(1-\Phi\left(u^{a-1 / 2)}\right) .\right.
\end{aligned}
$$

It then follows from (6.12), (6.13) and Fatou's lemma that

$$
\begin{aligned}
\liminf _{\epsilon \downarrow 0} \epsilon^{2(p a-1) /(2 a-1)} J(\epsilon ; p, \alpha) \\
\quad \geq 2 \int_{0}^{\infty} u^{p a-2}\left(1-\Phi\left(u^{(2 a-1) / 2}\right)\right) d u .
\end{aligned}
$$

To obtain the reverse inequality with $\lim$ inf replaced by $\lim$ sup, we let $0<\delta<1$ and define $X_{i}^{\prime}, X_{i}^{\prime \prime}$ by (6.6) with $K>0$ so chosen that (6.7) is satisfied and $0<\sigma \leq 1+\delta$, where $\sigma^{2}=E\left(X_{1}^{\prime}\right)^{2}$. Let $S_{n}^{\prime}=X_{1}^{\prime}+\cdots+X_{n}^{\prime}$, $S_{n}^{\prime \prime}=X_{1}^{\prime \prime}+\cdots+X_{n}^{\prime \prime}$ and define $J^{\prime}(\epsilon ; p, \alpha)$ for $S_{n}^{\prime}, J^{\prime \prime}(\epsilon ; p, a)$ for $S_{n}^{\prime \prime}$. Obviously $J(\epsilon ; p, \alpha) \leq J^{\prime}((1-\delta) \epsilon ; p, \alpha)+J^{\prime \prime}(\delta \epsilon ; p, \alpha)$. Using the inequality $(1.9)$ as in (6.8), we obtain that

$$
\epsilon^{2(p a-1) /(2 a-1)} J^{\prime \prime}(\delta \epsilon ; p, a)
$$

$$
\leq \xi_{p, a} \epsilon^{2(p a-1) /(2 a-1)} \sum_{n=1}^{\infty} n^{p a-2} P\left[\max _{1 \leq j \leq n}\left(s_{j}^{n} /(\delta \epsilon)\right) \geq n^{a}\right]
$$

$\leq \zeta_{p, a} \delta$, where $\xi_{p, a}$ and $\zeta_{p, a}$ depend only on $p$ and $\alpha$.

Choose $B \geq 1$ large enough that $u^{a}-(2 u)^{1 / 2}>1 / 2 u^{\alpha}$ for all $u \geq B$ and

$$
2 \int_{B}^{\infty} u^{p a-2}\left(1-\Phi\left(1 / 2 u^{a-1 / 2}\right)\right) d u \leq \delta .
$$

Let $\tilde{\epsilon}=(1-\delta) \epsilon / \sigma$. As in (6.12), we have

$$
\tilde{\epsilon}^{2(p a-1) /(2 a-1)} J^{\prime}((1-\delta) \epsilon ; p, a)
$$

$$
=\int_{0}^{\infty} u^{p a-2} P\left[\max _{j \leq u \tilde{\epsilon}^{-2 /(2 a-1)}}\left(S_{j}^{\prime} / \sigma\right) \geq \tilde{\epsilon}^{-1 /(2 a-1)} u^{\alpha}\right] d u .
$$


By the dominated convergence theorem,

$$
\begin{aligned}
\int_{0}^{B} u^{p a-2} P\left[\max _{j \leq u \tilde{\epsilon}^{-2 /(2 a-1)}}\left(s_{j}^{\prime} / \sigma\right) \geq \tilde{\epsilon}^{-1 /(2 a-1)} u^{a}\right] d u \\
\quad \rightarrow \int_{0}^{B} u^{p a-2} P\left[\max _{0 \leq t \leq 1} W(t) \geq u^{a-1 / 2}\right] d u \quad(\text { as } \epsilon \rightarrow 0) \\
\quad \leq 2 \int_{0}^{\infty} u^{p a-2}\left(1-\Phi\left(u^{(2 a-1) / 2}\right)\right) d u .
\end{aligned}
$$

Using the Lévy inequality [12, p. 248], we obtain for $u \geq B$,

$$
P\left[\max _{j \leq u \tilde{\epsilon}^{-2 /(2 a-1)}}\left(s_{j}^{\prime} / \sigma\right) \geq \tilde{\epsilon}^{-1 /(2 a-1)} u^{a}\right]
$$

$$
\leq 2 P\left[S_{\left[u \tilde{\epsilon}^{-2 /(2 a-1)]}\right.}^{\prime} / \sigma \geq 1 / 2 \tilde{\epsilon}^{-1 /(2 a-1)} u^{a}\right] \text {, since } u^{a}-(2 u)^{1 / 2}>1 / 2 u^{a} \text {. }
$$

We now apply an estimate, due to Esseen [6], to the above probability: Let $k$ be a positive integer such that $k-2>2(p a-1)$. Then there exist positive constants $c_{1}, c_{2}$ depending on the absolute moments $E\left|X_{1}^{\prime}\right|^{2}, E\left|X_{1}^{\prime}\right|^{3}$, $\cdots, E\left|X_{1}^{\prime}\right|^{k}$ such that for all $n=1,2, \cdots$ and all real $x$,

$$
\left|P\left[S_{n}^{\prime}<\sigma \sqrt{n x}\right]-\Phi(x)\right| \leq c_{1} n^{-1 / 2}\left(1+|x|^{3}\right) e^{-x^{2} / 2}+c_{2} n^{-(k-2) / 2}
$$

(cf. [6, pp. 73-76]). Set $n=\left[u \tilde{\epsilon}^{-2 /(2 a-1)}\right]$ for $u \geq B$. Then $n \geq 1$ if $\tilde{\epsilon}<1$. Therefore applying the above estimate, we have for $\tilde{\epsilon}<1$ and $u \geq B$,

$$
\begin{aligned}
P\left[S_{n}^{\prime} / \sigma \geq\right. & \left.1 / 2 \epsilon^{-1 /(2 a-1)} u^{a}\right] \leq P\left[S_{n}^{\prime} \geq 1 / 2 \sigma \sqrt{n u^{a-1 / 2}}\right] \\
\leq & 1-\Phi\left(1 / 2 u^{a-1 / 2}\right)+c_{1} n^{-1 / 2}\left(1+u^{3(a-1 / 2)}\right) \exp \left(-(1 / 8) u^{2 a-1}\right) \\
& +2^{(k-2) / 2} c_{2} \tilde{\epsilon}^{(k-2) /(2 a-1)} u^{-(k-2) / 2}
\end{aligned}
$$

Since $(k-2) / 2>p a-1$, it then follows from (6.19) that

$$
\underset{\epsilon ! 0}{\lim \sup } 2 \int_{B}^{\infty} P\left[s_{\left[u \tilde{\epsilon}^{\prime}-2 /(2 a-1)\right.}^{\prime} / \sigma \geq 1 / 2 \tilde{\epsilon}^{-1 /(2 a-1)} u^{a}\right] u^{p a-2} d u
$$

$$
\leq 2 \int_{B}^{\infty}\left(1-\Phi\left(1 / 2 u^{a-1 / 2}\right)\right) u^{p a-2} d u \leq \delta .
$$

It then follows from (6.15), (6.16), (6.17), (6.18) and (6.20) that 
$\lim \sup \epsilon^{2(p a-1) /(2 a-1)} J(\epsilon ; p ; a)$ $\epsilon \perp 0$

$$
\leq \zeta_{p, a} \delta+(\sigma /(1-\delta))^{2(p a-1) /(2 a-1)}\left\{2 \int_{0}^{\infty} u^{p a-2}\left(1-\Phi\left(u^{(2 a-1) / 2}\right)\right) d u+\delta\right\} .
$$

Since $\sigma \leq 1+\delta$ and $\delta$ is arbitrary, we therefore have

$$
\underset{\epsilon ! 0}{\lim \sup } \epsilon^{2(p a-1) /(2 a-1)} J(\epsilon ; p, \alpha) \leq 2 \int_{0}^{\infty} u^{p a-2}\left(1-\Phi\left(u^{(2 a-1) / 2}\right)\right) d u .
$$

In a similar way, we can obtain the asymptotic behavior of

$$
\int_{0}^{\infty} t^{p a-2} P\left[s_{t} \geq \epsilon t^{a}\right] d t
$$

as $\epsilon \downarrow 0$ given in (6.10). Finally, we note that

$$
P\left[\sup _{k \geq t} k^{-a} S_{k} \geq \epsilon\right] \geq P[T(\epsilon, \alpha) \geq t] \geq P\left[\sup _{k \geq t} k^{-a} S_{k}>\epsilon\right]
$$

and so (6.11) follows immediately from Theorem 7.

\section{REFERENCES}

1. L. E. Baum and M. Katz, Convergence rates in the law of large numbers, Trans. Amer. Math. Soc. 120 (1965), 108-123. MR 33 \#6679.

2. Y. S. Chow, Delayed sums and Borel summability for independent, identically distributed random variables, Bull. Inst. Math. Acad. Sinica 1 (1973), 207-220.

3. Y. S. Chow, H. Robbins and D. Siegmund, Great expectations: The theory of optimal stopping, Houghton-Mifflin, Boston, Mass., 1971.

4. J. L. Doob, Stochastic processes, Wiley, New York; Chapman \& Hall, L.ondon, 1953. MR 15, 445.

5. P. Erdös, On a theorem of Hsu and Robbins, Ann. Math. Statist. 20 (1949), 286-291. MR 11, 40.

6. C. -G. Esseen, Fourier analysis of distribution functions. A mathematical study of the Laplace-Gaussian law, Acta Math. 77. (1945), 1-125. MR 7, 312.

7. P. Hartman and A. Wintner, On the law of the iterated logarithm, Amer. J. Math. 63 (1941), 169-176. MR 2, 228.

8. C. S. Kao, On the time and the excess of linear boundary crossings of the sample sums, Ph. D. Thesis, Columbia University, New York, 1972.

9. J. Kiefer and J. Wolfowitz, On the characteristics of the general queueing process, with applications to random walk, Ann. Math. Statist. 27 (1956), 147-161. MR 17, 980.

10. J. F. C. Kingman, Some inequalities for the queue $G I / G / 1$, Biometrika 49 (1962), 315-324. MR 33 \#6720.

11. T. L. Lai, Limit theorems for delayed sums, Ann. Probability 2 (1974), 432-440.

12. M. Loève, Probability theory. Foundations. Random sequences, 3rd. ed., Van Nostrand, Princeton, N. J., 1963. MR 34 \#3596.

13. J. Marcinkiewicz et A. Zygmund, Quelques théorèmes sur les fonctions indépendantes, Studia Math. 7 (1938), 104-120.

14. H. Robbins and D. Siegmund, Boundary crossing probabilities for the Wiener process and sample sums, Ann. Math. Statist. 41 (1970), 1410-1429. MR 43 \#2796. 
15. H. Robbins, D. Siegmund and J. Wendel, The limiting distribution of the last time $S_{n} \geq n \epsilon$, Proc. Nat. Acad. Sci. U.S.A. 61 (1968), 1228-1230. MR 39 \#946.

16. F. Spitzer, A combinatorial lemma and its application to probability theory, Trans. Amer. Math. Soc. 82 (1956), 323-339. MR 18, 156.

17. A. Zygmund, Trigonometric series. Vols. 1, 2, 2nd ed., Cambridge Univ. Press, New York, 1959. MR 21 \#6498.

18. L. Baum, On convergence to $+\infty$ in the law of large numbers, Ann. Math. Statist. 34 (1963), 219-222. MR 26 \#799.

19. D. W. Müller, Verteilungs-Invarianzprinzipien für das starke Gesetz der grossen Zahl, Z. Wahrscheinlichkeitstheorie und Verw. Gebiete 10 (1968), 173-192. MR 38 \#753.

DEPARTMENT OF MATHEMATICAL STATISTICS, COLUMBIA UNIVERSITY, NEW YORK, NEW YORK 10027 